\section{Transmembrane trajectories}

Many proteins are inserted into the cell membrane by translocons, or multiprotein assemblies that recognize nascent polypeptides. However, studying translocon function is complicated by the fast kinetics of transport, a paucity of methods to determine which portion of a new protein is in contact with the translocon, and the intrinsic challenges of working with the heterogeneous cell membrane and hydrophobic protein substrates. von Heinje and colleagues had previously developed a method to determine the energetics of inserting different amino acids into the center of a known transmembrane helix, but it wasn't obvious how these energetics might translate to placement at other portions of the helix. Now Hessa et al. have used this methodology to conduct a systematic scan of all residues at all transmembrane positions to quantitatively determine that the effect of weakly polar and apolar residues is independent of position. However, for charged and polar residues, as well as proline, the anisotropic environment of the membrane causes a significant shift in the energetics of incorporation. Further, with the exception of proline, the introduction of multiple amino acids could be estimated using a simple additive model. This finding allowed the authors to quantitate the energetics of natural proteins; surprisingly, they discovered that the membrane insertion of a significant portion of known transmembrane regions is unfavorable, thus requiring these helices to rely on more hydrophobic neighboring helices or interhelical interactions to generate stability in the membrane. (Nature 450, 1026-1030, 2007)

\section{Selectivity gets a score}

\begin{abstract}
Although it is well established that kinase inhibitors can hit multiple targets and that even clinically approved kinase inhibitors can be relatively promiscuous, kinase inhibitor selectivity has not been comprehensively investigated. Karaman et al. have now determined the dissociation constant $\left(K_{\mathrm{d}}\right)$ for the binding of 38 kinase inhibitors to 317 kinases, which covers more than $50 \%$ of the human protein kinome. To facilitate a
\end{abstract} quantitative comparison of inhibitor selectivity, the authors developed 'selectivity scores', which are calculated by dividing the total number of kinase inhibitors found to bind an inhibitor below a threshold $K_{\mathrm{d}}$ by the total number of kinases tested. As a step toward estimating the biologically relevant level of selectivity, the authors then determined the number of 'off-target' kinases that were inhibited with a $K_{\mathrm{d}}$ within 10 -fold of the binding affinity of the intended target. Although many kinase inhibitors were relatively selective by this measure, there were a number of compounds, including sorafenib and desatinib, that bound other kinases with an affinity similar to that of their primary target. These results provide an important step forward in quantitating in vitro kinase inhibitor specificity, and they also raise a number of new questions, including what is the impact of different selectivity scores in a cellular and clinical setting and how can quantitative measures of selectivity be used to optimize the development of new kinase inhibitors. (Nat. Biotechnol., published online 8 January 2008, doi:10.1038/ nbt1358)

Written by Mirella Bucci, Catherine Goodman, Joanne Kotz \& Terry L. Sheppard

\section{Strictly strictosidine}

The Pictet-Spengler reaction, a widely used synthetic transformation, finds its origins in the biosynthesis of many plant alkaloids. For example, strictosidine synthase catalyzes the biosynthesis of the monoterpene indole

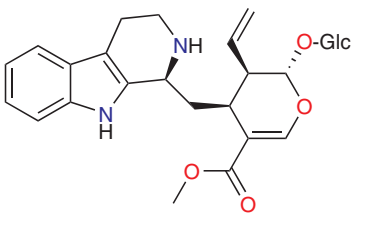
alkaloid strictosidine in two steps: the formation of an iminium ion by condensation of tryptamine with the aldehyde moiety of secologanin, and an intramolecular electrophilic aromatic substitution reaction to produce the tricyclic $\beta$-carboline skeleton. Crystallographic and biochemical studies of strictosidine synthase have suggested that a glutamate residue (Glu309) is important for catalysis, but the detailed mechanism has not yet been elucidated. Maresh et al. now use kinetic, structural and computational methods to understand the solution-phase Pictet-Spengler reaction and the mechanism of strictosidine synthase. The bell-shaped $\mathrm{pH}$-rate profile of the enzymatic reaction revealed two catalytically important ionizable groups, one of which was attributed to Glu309. The enzymatic and solutionphase reactions exhibited a significant primary kinetic isotope effect $(>2.0)$ with a deuterated tryptamine substrate, which suggests that the final rearomatization step is rate limiting for the overall reaction. For this to be true, formation of the iminium ion and the subsequent cyclization reaction would need to be fast and reversible. Based on this assumption and additional studies, the authors argue that strictosidine synthase has two major roles: to position substrates, thereby controlling the diastereoselectivity of the reaction, and to use Glu309 for general acid catalysis of iminium ion formation. The study reveals new features of catalysis by strictosidine synthase and underscores the important similarities and contrasts between enzymatic reactions and their solution counterparts. (J. Am. Chem. Soc., published online 15 December 2007, doi:10.1021/ja077190z)

\section{Ha-ha-ha}

The trinucleotide sequences that specify the genetic code can be manipulated to generate polypeptide chains that contain various noncanonical amino acids. In vivo, this can be done using nonsense or frameshift suppressor aminoacyl-tRNAs (aa-tRNAs) as adaptors. In vitro, the components of a translation system can be manipulated to exclude selected aminoacyl-tRNA synthetases (ARSs) and include recombinant versions designed to incorporate non-natural amino acids or other nonproteinogenic moieties, provided they are able to form a ribosome-mediated amide or ester bond. $\alpha$-hydroxy acids, the backbone of polyesters, meet these criteria, but complex polyesters and other polymers with exclusively nonpeptide backbones have not been generated in this way because straightforward methods have not been available. Ohta et al. now use artificially evolved ribozymes called flexizymes to charge seven different $\alpha$-hydroxy acids (ha) onto tRNAs, each bearing different anticodons. Such ha-tRNAs could then be added to an Escherichia coli cell-free translation system called wPURE, in which amino acids and ARSs are withheld to exclude incorporation of the corresponding natural amino acids. They found that they could apply this strategy to any of the 20 aa-tRNAs and could strictly control the length of the polyesters based on the mRNA template sequence added to the reactions to synthesize 9 different polyesters with lengths varying from 3 to $12 \alpha$-hydroxy acid units. This strategy could be used to study polyester and polyester-polypeptide polymers engineered with a wide variety of side chains having strictly controlled sequences and lengths. (Chem. Biol. 14, 1315-1322, 2007)

$M B$ 